\title{
MDEntropy: Information-Theoretic Analyses for Molecular Dynamics
}

\section{Carlos X. Hernández ${ }^{1}$ and Vijay S. Pande ${ }^{1}$}

DOI: $10.21105 /$ joss. 00427

1 Stanford University

\section{Software}

- Review ¿t

- Repository ca

- Archive [T

\section{Licence}

Authors of JOSS papers retain copyright and release the work under a Creative Commons Attribution 4.0 International License (CC-BY).

\section{Summary}

MDEntropy is a Python package for information-theoretic (IT) analysis of data generated from molecular dynamics simulations. While correlation studies have long been of interest to the molecular dynamics (MD) community (McCammon, Gelin, and Karplus 1977, McClendon et al. (2009)), IT tools to analyze MD trajectories have been much less developed. MDEntropy seeks to fill this niche by providing an easy-to-use Python API that works seamlessly with other Python packages, such as mdtraj, msmbuilder, and numpy (R. T. McGibbon et al. 2015, Walt, Colbert, and Varoquaux (2011), M. P. Harrigan et al. $(2017))$.

Functionality in MDEntropy is centered around mdtraj trajectories and the statistical tools available in msmbuilder. Leveraging these tools allows for statistically robust analyses of many IT estimators across a variety of biomolecular feature-spaces (Schreiber 2000, Kraskov, Stögbauer, and Grassberger (2004)).

MDEntropy is actively developed and maintained by researchers at Stanford University. Source code for MDEntropy is hosted on GitHub and is continuously archived to Zenodo (Hernández and Pande 2017). Full documentation, including Jupyter Notebook tutorials, can be found at http://msmbuilder.org/mdentropy.

\section{References}

Harrigan, Matthew P., Mohammad M. Sultan, Carlos X. Hernández, Brooke E. Husic, Peter Eastman, Christian R. Schwantes, Kyle A. Beauchamp, Robert T. McGibbon, and Vijay S. Pande. 2017. "MSMBuilder: Statistical Models for Biomolecular Dynamics." Biophysical Journal 112 (1): 10-15. doi:10.1016/j.bpj.2016.10.042.

Hernández, Carlos X., and Vijay S. Pande. 2017. "Msmbuilder/Mdentropy: V0.3." doi:10.5281/zenodo.1000997.

Kraskov, Alexander, Harald Stögbauer, and Peter Grassberger. 2004. "Estimating mutual information." Physical Review E 69 (6). American Physical Society: 066138. doi:10.1103/PhysRevE.69.066138.

McCammon, J. Andrew, Bruce R. Gelin, and Martin Karplus. 1977. "Dynamics of folded proteins." Nature 267 (5612). Nature Publishing Group: 585-90. doi:10.1038/267585a0.

McClendon, Christopher L, Gregory Friedland, David L Mobley, Homeira Amirkhani, and Matthew P Jacobson. 2009. "Quantifying Correlations Between Allosteric Sites in Thermodynamic Ensembles." Journal of Chemical Theory and Computation 5 (9): 24862502. doi:10.1021/ct9001812.

McGibbon, Robert T, Kyle A Beauchamp, Matthew P Harrigan, Christoph Klein, Ja- 
son M Swails, Carlos X Hernández, Christian R Schwantes, Lee-Ping Wang, Thomas J Lane, and Vijay S Pande. 2015. "MDTraj: A Modern Open Library for the Analysis of Molecular Dynamics Trajectories." Biophysical Journal 109 (8). Elsevier: 1528-32. doi:10.1016/j.bpj.2015.08.015.

Schreiber, Thomas. 2000. "Measuring Information Transfer." Physical Review Letters 85 (2): 461-64. doi:10.1103/PhysRevLett.85.461.

Walt, Stéfan van der, S Chris Colbert, and Gael Varoquaux. 2011. "The NumPy Array: A Structure for Efficient Numerical Computation." Computing in Science $\mathcal{E}$ Engineering 13 (2): 22-30. doi:10.1109/MCSE.2011.37. 